\title{
Radiation Hardness of a Large Area CMOS Active Pixel Sensor for Bio-medical applications
}

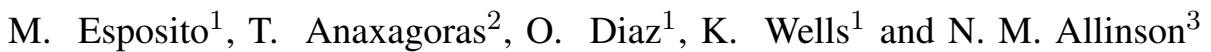

\begin{abstract}
A wafer scale CMOS Active Pixel Sensor has been designed employing design techniques of transistor enclosed geometry and $\mathrm{P}+$ doped guard rings to offer ionizing radiation tolerance. The detector was irradiated with $160 \mathrm{kVp} \mathrm{X}$-rays up to a total dose of $94 \mathrm{kGy}(\mathrm{Si})$ and remained functional. The radiation damage produced in the device has been studied, resulting in a dark current density increase per decade of $96 \pm 5 \mathrm{pA} / \mathrm{cm}^{2} / \mathrm{decade}$ and a damage threshold of $204 \mathrm{~Gy}(\mathrm{Si})$. The damage produced in the detector has been compared with a commercially available CMOS APS, showing a radiation tolerance about 100 times higher. Moreover Monte Carlo simulations have been performed to evaluate primary and secondary energy deposition in each of the detector stages.
\end{abstract}

Index Terms-Radiation hardness, CMOS, APS, Large area image sensors, semiconductor devices, Monte Carlo simulations, Geant4, Fluorescence X-rays.

\section{INTRODUCTION}

$\mathbf{I}$ $\mathrm{N}$ many biomedical imaging applications there is a strong demand for large area sensors. For the last two decades amorphous Silicon and amorphous Selenium based flat panel imagers (FPI) have represented the detector of choice in digital medical imaging, mainly because they can be easily fabricated on a large area with a relatively low cost technology, derived from the consumer based flat panel display technology [1].

Even so FPIs suffer significant drawbacks, which impact on imaging performance, such as large pixels, high noise $(>1000$ $\left.e^{-}\right)$, low frame rate and image lag [2]. In this scenario CMOS Active Pixel Sensors (APS) [3] have gained popularity having demonstrated capability to overcoming such issues, offering a lower noise $\left(60-150 e^{-}\right)$, a pixel pitch in the order of $25-50 \mu \mathrm{m}$, a high frame rate based on a true random access via column parallel readout and absence of image artefacts [4], [5]. These advantages, together with low power consumption and potential for a low cost and fast scaling technology (based on standard consumer-based CMOS fabrication), have made CMOS APSs a valuable alternative in the bio-medical imaging field.

Furthermore, recent developments in photo-lithographic techniques [6] have made available the realization of large area devices integrated onto an eight inches silicon wafers to create a contiguous sensor array scalable up to the wafer size, i.e. 13 $\mathrm{cm} \times 13 \mathrm{~cm} \mathrm{[7].}$

In order for CMOS APSs to impact upon the medical ionzing

\footnotetext{
${ }^{1}$ Centre for Vision, Speech and Signal Processing, Faculty of Engineering and Physical Sciences, University of Surrey, Guildford GU2 7XH,U.K. (email:m.esposito@surrey.ac.uk ).

${ }^{2}$ ISDI Ltd (Image sensor design and innovation), Oxford, OX4 1YZ, U.K

${ }^{3}$ School of Computer Science, University of Lincoln, Lincoln, LN6 7TS, U.K.
}

applications area, then it needs to demonstrate a significant radiation hardness. Several investigations have been carried out to assess the radiation tolerance of CMOS devices [8], [9] and to propose new design techniques to enhance this performance [10].

In this paper we propose a novel wafer scale CMOS APS, developed in the framework of the Multidimensional Integrated Intelligent Imaging Plus (MI-3 Plus) consortium. This detector has been designed for bio-medical applications and offers a high radiation hardness-by-design [10]. The radiation hardness of this detector has been characterized and the results have been compared with a commercial CMOS APS for radiology applications.

\section{Materials AND MethodS}

\section{A. The DynAMITe detector}

The APS presented here, named the Dynamic range Adjustable for Medical Imaging Technology or DynAMITe, was constructed in a $0.18 \mu \mathrm{m}$ CMOS process by reticule stitching technique for a total active area of $12.8 \mathrm{~cm} \times 13.1 \mathrm{~cm}$. A picture of the wafer from which the DynaAMITe sensor was diced is shown in Fig. 1.

The DynAMITe pixel array consists of two different size diodes meshed in the same pixel matrix, thus realizing two imagers in one. The detector consists of fine-pitch grid diodes, offering intrinsic low noise and high spatial resolution, and a large-pitch grid diodes, offering a high dynamic range. Both grids are geometrically superimposed. Thus each cell of the DynAMITe matrix is fitted with multiple diodes: four diodes of small size (50 $\mu \mathrm{m}$ side), named Sub-Pixels, and one diode of large size (100 $\mu \mathrm{m}$ side), named Pixel. The whole matrix comprises $1312 \times 1280$ Pixels and $2624 \times 2560$ Sub-Pixels. A more detailed description of the pixel architecture, the read out modalities and electro-optical performance are reported in [5].

\section{B. The radiation hard design of the DynAMITe detector}

The DynAMIte detector has been designed according to the radiation hardness-by-design methodology.

In fact all the in-pixel transistors have been designed with source and drain physically enclosed using an Enclosed Layout Geometry (ELG) [10], [11] in order to reduce the edgeleakage, which is generated in the transition area between the thin gate oxide and the thick field oxide, used to produce transistor-by-transistor insulation, after exposure to radiation. $\mathrm{P}^{+}$doped guard rings have been added in each pixels to prevent radiation induced inter-device leakage current. 


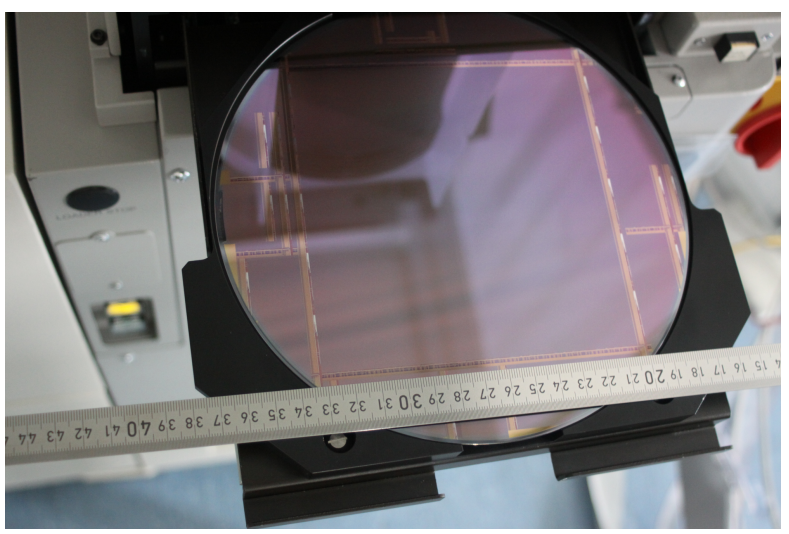

Fig. 1. DynAMITe chip wafer. The boundaries of the chip region are visible with the ruler denoting the $12.8 \mathrm{~cm}$ edge dimension.

Moreover the standard sub-micrometer process $(0.18 \mu \mathrm{m})$ used guarantees a thin field oxide, comparable with the tunnelling length for holes in $\mathrm{SiO}_{2}$. This increases the probability for holes to tunnel out of the gate oxide avoiding the formation of trapped charge at the interface $\mathrm{SiO}_{2}-\mathrm{Si}$, responsible for threshold voltage shift in NMOS transistors exposed to ionizing radiation.

\section{Irradiation experimental set-up}

The DynAMITe detector has been exposed to an X-ray field (W anode, $160 \mathrm{kVp}, 0.5 \mathrm{~mm} \mathrm{Cu}$ filtration) up to a cumulative dose of $94 \mathrm{kGy}(\mathrm{Si})$. An average of 15 hours of exposure per day was performed while the detector was biased, reset and readout in order to recreate actual operational conditions.

The detector was provided with a scintillator $(140 \mu \mathrm{m}$ thick $\mathrm{Gd}_{2} \mathrm{O}_{2} \mathrm{~S}: \mathrm{Tb}$ ) and a $3 \mathrm{~mm}$ Fiber Optic Plate (FOP). Performance parameters such as dark current, offset, gain and dynamic range have been evaluate at each exposure step for two separate region of interest (ROIs) of approximately $1 \mathrm{~cm}^{2}$ area each. The rest of the detector was covered with a $2 \mathrm{~mm}$ thick Tungsten shiel.

The dark current was measured by subtraction of two dark images, one with a long integration time and one with a short integration time, in order to correct for the remaining Fixed Pattern Noise. The dark current measured with this procedure was converted into dark current density using the conversion gain of the sensor calculated at each exposure step.

A commercial APS designed for bio-medical applications, has been irradiated in the same test experiment up to a cumulative dose of $0.8 \mathrm{kGy}$ (Air Kerma) when it showed loss of light sensitivity and pixel resolution.

The maximum delivered dose in this experiment $(94 \mathrm{kGy}(\mathrm{Si})$ ) represents the highest deliverable dose within the time allocated for this experiment.

\section{Monte Carlo simulations}

In order to evaluate energy absorption across all the layers constituting the detection system, Monte Carlo simulations were performed using the GEANT4 toolkit[12] and the Livermore low-energy electromagnetic models [13]. The detection
TABLE I

SiMULATED GEOMETRY

\begin{tabular}{|c|c|c|c|c|}
\hline Layer & Thickness & Length & Width & Composition \\
\hline Shield & $2 \mathrm{~mm}$ & $7 \mathrm{~cm}$ & $3 \mathrm{~cm}$ & $\mathrm{~W}$ \\
\hline $\begin{array}{l}\text { Protective layer } \\
\text { (Scintillator) }\end{array}$ & $6 \mu \mathrm{m}$ & $7 \mathrm{~cm}$ & $7 \mathrm{~cm}$ & PET \\
\hline $\begin{array}{l}\text { Gadox } \\
\text { (Scintillator) }\end{array}$ & $140 \mu \mathrm{m}$ & $7 \mathrm{~cm}$ & $7 \mathrm{~cm}$ & $\mathrm{Gd}_{2} \mathrm{O}_{2} \mathrm{~S}: \mathrm{Tb}$ \\
\hline $\begin{array}{l}\text { Plastic base } \\
\text { (Scintillator) }\end{array}$ & $250 \mu \mathrm{m}$ & $7 \mathrm{~cm}$ & $7 \mathrm{~cm}$ & Perspex \\
\hline FOP & $3 \mathrm{~mm}$ & $7 \mathrm{~cm}$ & $7 \mathrm{~cm}$ & $\begin{array}{r}33 \% \mathrm{SiO}_{2} \\
30 \% \mathrm{PbO} \\
12 \% \mathrm{La}_{2} \mathrm{O}_{3} \\
6 \% \mathrm{Al}_{2} \mathrm{O}_{3} \\
6 \% \mathrm{~B}_{2} \mathrm{O}_{3} \\
1.5 \% \mathrm{ZrO}_{2} \\
0.6 \% \mathrm{BaO}\end{array}$ \\
\hline $\begin{array}{l}\text { Silicon Nitride } \\
\text { (Detector) }\end{array}$ & $0.45 \mu \mathrm{m}$ & $7 \mathrm{~cm}$ & $7 \mathrm{~cm}$ & $\mathrm{Si}_{3} \mathrm{O}_{4}$ \\
\hline $\begin{array}{l}\text { Silicon Oxide } \\
\text { (Detector) }\end{array}$ & $0.05 \mu \mathrm{m}$ & $7 \mathrm{~cm}$ & $7 \mathrm{~cm}$ & $\mathrm{SiO}_{2}$ \\
\hline $\begin{array}{l}\text { Epitaxial layer } \\
\text { (Detector) }\end{array}$ & $12 \mu \mathrm{m}$ & $7 \mathrm{~cm}$ & $7 \mathrm{~cm}$ & $\mathrm{Si}$ \\
\hline $\begin{array}{l}\text { Substrate } \\
\text { (Detector) }\end{array}$ & $713 \mu \mathrm{m}$ & $7 \mathrm{~cm}$ & $7 \mathrm{~cm}$ & $\mathrm{Si}$ \\
\hline
\end{tabular}

system was schematized as constituted of the layers reported in table I.

A number of 10 simulations of $10^{9}$ photons, randomly extracted from the relevant calculated X-ray spectrum [14] (W anode, $160 \mathrm{kVp}, 0.5 \mathrm{~mm} \mathrm{Cu}$ filtration), was performed. Energy deposition in each of the layers constituting the detection system was scored as fraction of the integral energy emitted by the source. Secondary radiation generated as fluorescence Xray in the shield, scintillator and FOP was taken into account and separately analyzed per generating chemical element.

Furthermore a second simulation was run to calculate Air Kerma (AirKerma ${ }^{\text {sim }}$ ) at the entrance window of the detector. Considering energy deposition evaluated in the $i$-th detection layer as function of AirKerma ${ }^{\text {sim }}$ $\left(E_{\text {dep }}^{i}\left(\right.\right.$ AirKerma $\left.\left.{ }^{\text {sim }}\right)\right)$ and the Air Kerma experimentally measured at each irradiation step $j$, facilitated calculation of the dose delivered to the detector layer $i$ at the irradiation step j $D_{i}(j)$.

\section{RESULTS}

\section{A. Energy absorption and fluorescence}

Energy absorption in the detector layers reported in table I is shown in fig. 2 as percentage of the integral energy emitted by the source. The maximum of energy deposition occurs for shield (18\%), scintillator (13\%) and FOP (31.5\%) as expected because of their thickness and atomic number. The percentage of energy absorbed in silicon (including silicon nitrite and oxide) is $0.23 \%$.

Contribution to the total energy absorbed in silicon from the $\mathrm{X}$-ray fluorescence component is shown in fig. 3, displaying the percentage of energy deposited in each of the detector layers, due to fluorescence generated in the shield, scintillator and FOP. The main contribution to fluorescence is represented by the FOP (between $25-18 \%$ ), because of the relatively high $\mathrm{Z}$ elements of which it is made $(\mathrm{Pb}, \mathrm{La}, \mathrm{Zr}, \mathrm{Ba})$, whereas the scintillator and shield contributes for a fraction evaluated in 


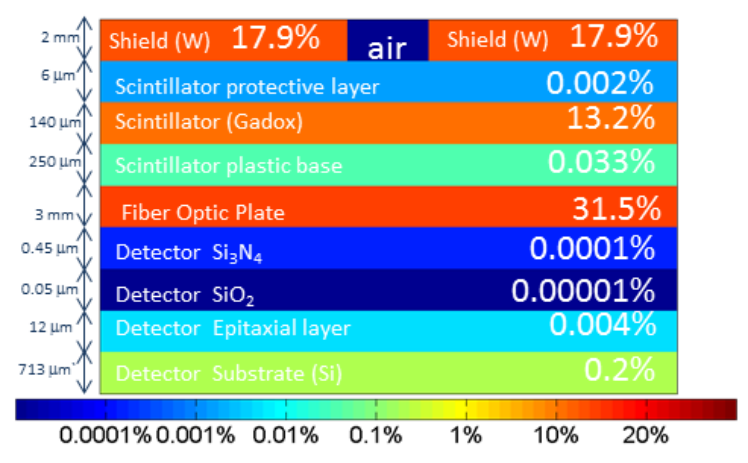

Fig. 2. Energy absorption in the detector layers. The logarithmic color scale represents the energy absorbed as percentage of the integral energy emitted by the source. Figure is not to scale.

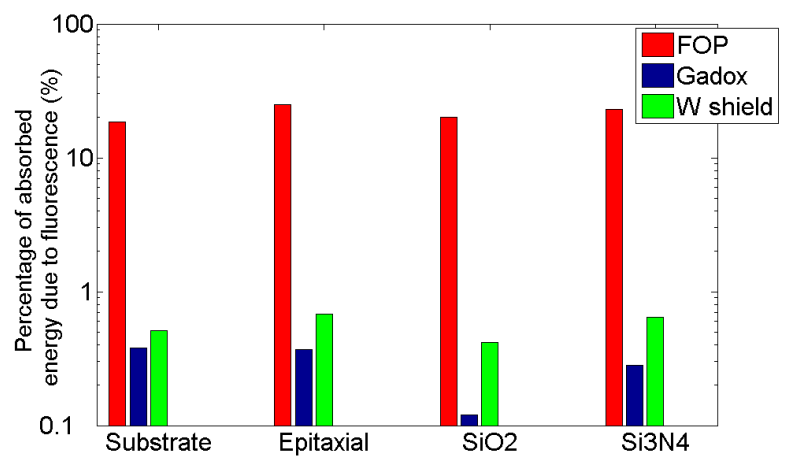

Fig. 3. Contribution to the total energy absorbed in silicon from fluorescence $\mathrm{X}$-rays produced in shield, scintillator and FOP.

the range $0.1-0.7 \%$.

Fig. 4 shows the contribution to the energy absorbed in the detection layer due to fluorescence X-rays generated in the FOP per chemical element. Lead represents the first source of fluorescence in the FOP (71-65\%) because of its relatively high abundance (30\%), followed by Lanthanum (20-33\%) present in the FOP in a percentage of $12 \%$. Barium and Zirconium offer a smaller contribution (2-0.4\%) depending on their smaller abundance in the FOP. Even so L-shell fluorescence X-rays due to these two elements are in the range $2-6 \mathrm{keV}$, implying a high cross section for such photons.

\section{B. Comparison with a commercial device}

Figs. 5 and 6 show comparative data for leakage current and dynamic range measured for both the DynAMITe detector and a commercial device.

Leakage current increases exponentially for the commercial device (Comm. dev.) up to a dose of $700 \mathrm{~Gy}$, where loss of light sensitivity and pixel resolution occurs (dotted line in Fig. 5 and 6). At this dose the relative increase in dark current for the DynAMITe detector is lower than $150 \%$ (100 times lower than the increase observed in the commercial device) and is almost constant up to the maximum delivered dose $(1.8 \mathrm{kGy}$ (Si)).

The residual dynamic range, calculated with the respect to the

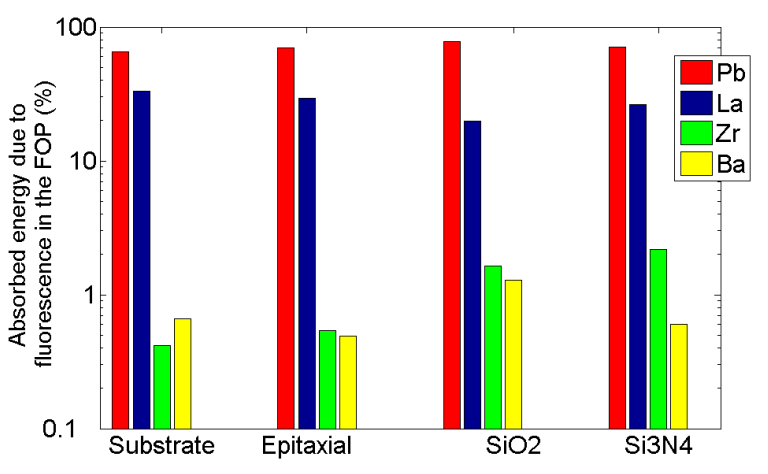

Fig. 4. Contribution to the energy absorbed in the detection layer due to fluorescence X-rays generated in the FOP per chemical element

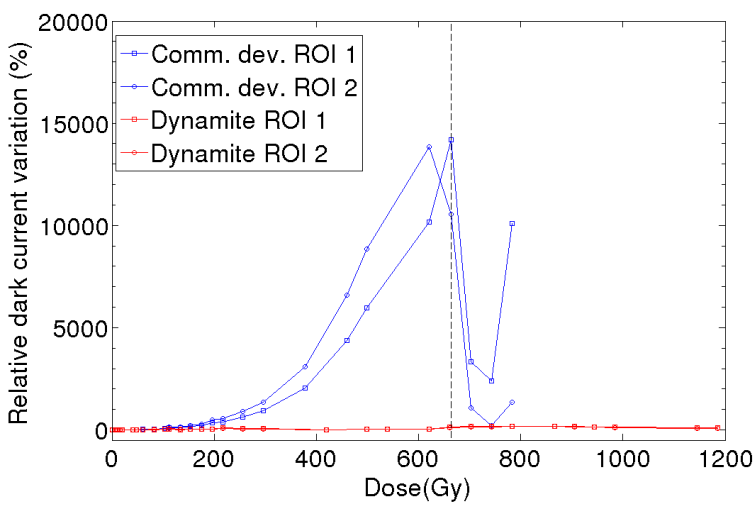

Fig. 5. Relative increase in dark current reported for the DynAMIte detector and a commercial device (Comm. dev.) in both the exposed ROIs.

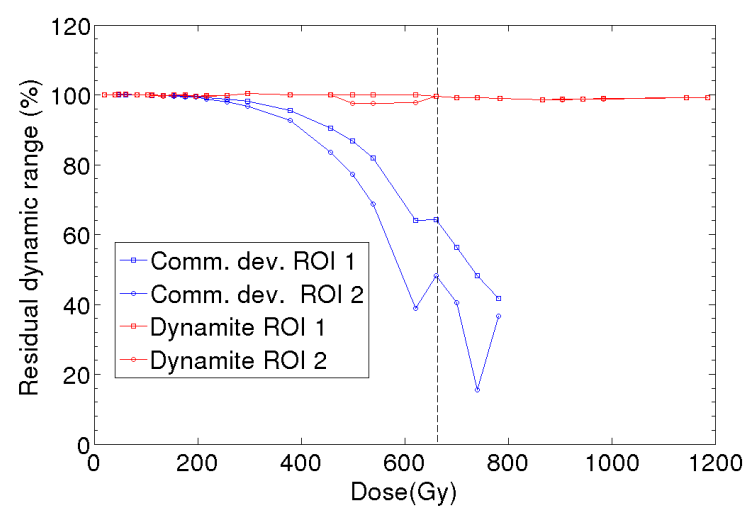

Fig. 6. Residual dynamic range reported for the DynAMIte detector and a commercial device (Comm. dev.) in both the exposed ROIs..

dynamic range of the unexposed sensors, is shown in Fig. 6. The DynAMIte detector exhibits a residual dynamic range of about $99 \%$ of the pre-irradiation one up to $1.2 \mathrm{kGy}$ Air Kerma $(1.8 \mathrm{kGy}(\mathrm{Si}))$, whereas the commercial device falls below the failure limit of $50 \%$ at $650 \mathrm{~Gy}$. The DynAMITe detector has been further irradiated up to a dose of $94 \mathrm{kGy}(\mathrm{Si})$ without showing any functionality issues with a residual dynamic range of $88 \%$ of the pre-irradiated one. 


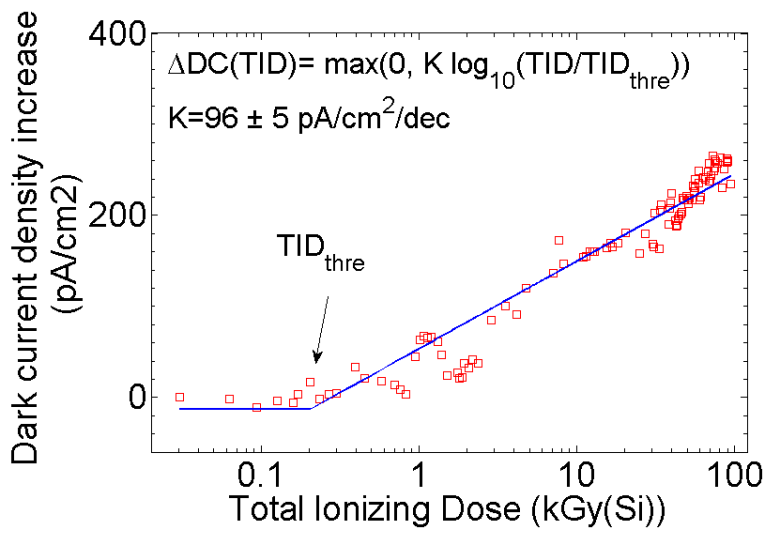

Fig. 7. Dark current density increase as function of the total ionizing dose to silicon (semi-logarithmic scale).

\section{Evaluation of the radiation damage}

Dark current density has been measured at each irradiation step to evaluate radiation induced damage. The pre-irradiation dark current density of the DynAMITe detector is $59 \mathrm{pA} / \mathrm{cm}^{2}$, a figure which increases up to $293 \mathrm{pA} / \mathrm{cm}^{2}$ at the maximum delivered dose of $94 \mathrm{kGy}(\mathrm{Si})$.

Fig. 7 shows the dark current density increase as function of the total ionizing dose to silicon $D_{S i}(j)$, calculated from Monte Carlo simulations, on a semi-logarithmic scale. The dark current increase due to the total ionizing dose TID $(\Delta C(T I D))$ has been fitted with the empirical formula [9]:

$$
\Delta C(T I D)=\max \left\{0, \log _{10} \frac{T I D}{\text { TID thre }}\right\}
$$

where $T I D_{\text {thre }}$ is the total ionizing dose threshold, necessary to observe a dark current density increase, and $K$ is the dark current increase per decade. Data of fig. 7 have been fitted with this function resulting in a radiation damage threshold TI $D_{\text {thre }}=204 \mathrm{~Gy}(\mathrm{Si})$ and a dark current density increase rate $K=96 \pm 5 \mathrm{pA} / \mathrm{cm}^{2} / \mathrm{dec}$.

Dark current density histograms are displayed in fig. 8 for six delivered doses $(0,0.6,1,10,50,94 \mathrm{kGy}(\mathrm{Si}))$. Two different phenomena are observable in the plot: a shift of the dark current density distribution towards higher current values, and a broadening of the distributions. After Gaussian fit of these distributions, the coefficient of variations, namely the ratio between the standard deviation and the mean of the Gaussian distributions, is plotted in fig. 9 for the six dose level evaluated. The highest coefficient of variation occurs for the pre-irradiation distribution (18\%), whereas this value ranges between 8 and $11 \%$ for all other dose levels implying no significant spatial non-uniformity arising from radiation damage produced up to $94 \mathrm{kGy}(\mathrm{Si})$.

\section{Conclusions}

A novel wafer scale CMOS APS has been presented as ionizing radiation tolerant detector up to at least a dose of $94 \mathrm{kGy}(\mathrm{Si})$, highest dose deliverable within the time allocated for the experiment. Dark current density increase and damage threshold have been evaluated as function of the total dose

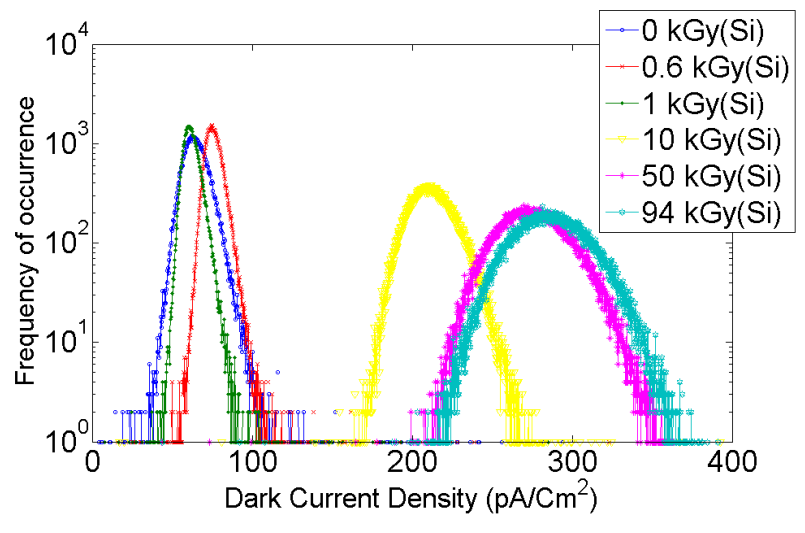

Fig. 8. Dark current density histogram at six total ionizing doses.

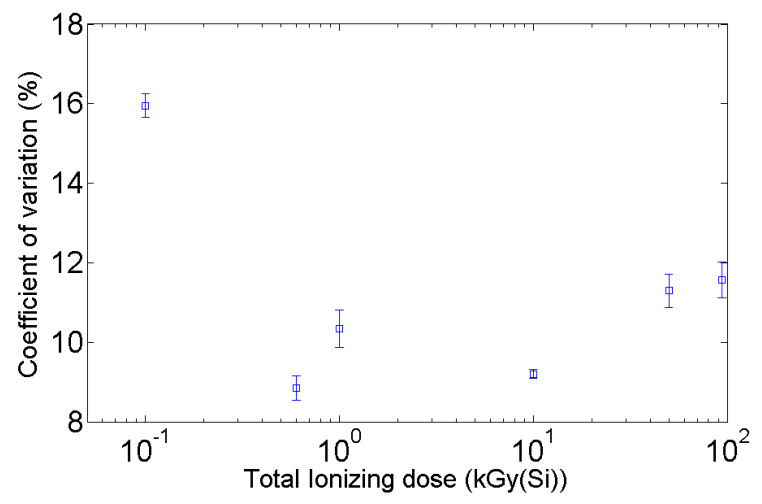

Fig. 9. Coefficient of variation of the dark current density distributions evaluated in fig. 8 .

delivered. The radiation damage has been compared against damage produced in a commercially available CMOS APS, showing a radiation tolerance at least 100 times higher. Monte Carlo simulations have been performed for evaluation of primary and secondary energy deposition, offering information on potential optimization of the detection system.

\section{ACKNOWLEDGMENT}

This work was supported by the RC-UK Multidimensional Integrated Intelligent Imaging Plus (MI-3 Plus) programme (EPSRC Ref. EP/G037671/1)

\section{REFERENCES}

[1] J. A. Rowlands and J. Yorkston, "Flat Panel Detectors for Digital Radiography ",Handbook of Medical Imaging, Volume 1 SPIE Press, Bellingham, WA, 2000

[2] R. L. Weisfield and N. R. Bennett, "Electronic noise analysis of a 127 m pixel TFT/photodiode array ", Proc. Med. Imag.: Phys. Med. Imag., 2001, pp. 209218

[3] F. R. Fossum, "CMOS image sensors: Electronic camera-on-a-chip ", IEEE Trans. Electr. Dev., 44 1689-1698 (1997)

[4] C. D. Arvanitis et al., "Empirical electro-optical and x-ray performance evaluation of CMOS active pixel sensor for low dose, high resolution x-ray medical imaging ", Med. Phys., 34 (12) 4612-4625, 2007

[5] M. Esposito et al., "DynAMITe: a wafer scale sensor for biomedical applications ", Journ. Instr., 6 C12064, 2011.

[6] D. Cohen et al., "Stitching design rules for forming interconnect layers", US patent 6225013, 1999.

[7] D. Scheffer, "Wafer scale active pixels CMOS image sensor for generic x-ray radiology ", Proc. SPIE, vol.6510, p. 65100O, 2007. 
[8] E. Eid, "Design and characterization of ionizing radiation tolerant CMOS APS image sensors up to $30 \mathrm{Mrd}(\mathrm{Si})$ total dose ", IEEE Trans. Nucl. Sc, 48(6), 2001.

[9] J. Bogaerts et al., "Total Dose and Displacement Damage Effects in a Radiation-Hardned CMOS APS", IEEE Trans. Elec. Dev., 50(1) 84-90 2003.

[10] R. C. Lacoe, "Improving integrated circuit performance through the application of hardness-by-design methodology", IEEE Trans. Nucl. Sc., 55(4), 2009.

[11] W. Snoeys et al., "Layout techniques to enhance the radiation tolerance of standard CMOS technologies demonstrated on a pixel detector readout chip ", Nucl. Instrum. and Meth. A, 439 (23), 349360, 2000

[12] S. Agostinelli et al., "Geant4 - A simulation toolkit", Nucl. Instrum. and Meth. A, 506 (3) 250-303 2003.

[13] G. A. P. Cirrone et al., "Validation of the Geant4 electromagnetic photon cross-sections for elements and compounds", Nucl. Instrum. and Meth. A, 618 315-322 (2010).

[14] G. Poludniowski et al., "SpekCalc: a program to calculate photon spectra from tungsten anode x-ray tubes", Phys. Med. Biol., 554 N433 2009. 